\title{
The human herpesvirus 8 chemokine receptor vGPCR triggers autonomous proliferation of endothelial cells
}

\author{
Marcos G. Grisotto, ${ }^{1}$ Alexandre Garin, ${ }^{1}$ Andrea P. Martin, ${ }^{1}$ Kristian K. Jensen, ${ }^{1}$ \\ PokMan Chan, ${ }^{2}$ Stuart C. Sealfon, ${ }^{2}$ and Sergio A. Lira ${ }^{1}$ \\ ${ }^{1}$ Immunobiology Center and ${ }^{2}$ Department of Neurology and Center for Translational Systems Biology, \\ Mount Sinai School of Medicine, New York, New York, USA.
}

\begin{abstract}
We have used a novel conditional transgenic system to study the mechanisms of angioproliferation induced by viral G protein-coupled receptor (vGPCR), the constitutively active chemokine receptor encoded by human herpesvirus 8 (HHV8, also known as Kaposi sarcoma herpesvirus). Using this system, we were able to control temporal expression of vGPCR and to monitor its expression in situ via the use of the surrogate marker LacZ. Upon treatment with doxycycline (DOX), cells expressing vGPCR and LacZ (vGPCR/LacZ ${ }^{+}$cells) progressively accumulated in areas where angioproliferation was observed. Sorted $\mathrm{vGPCR} / \mathrm{LacZ}^{+}$cells from angiogenic lesions expressed markers characteristic of endothelial progenitor cells, produced angiogenic factors, and proliferated in vitro. Prolonged treatment of transgenic mice with DOX led to development of tumors in the skin of ears, tail, nose, and paws. vGPCR/LacZ ${ }^{+}$cells were frequent in early lesions but scarce within these tumors. Finally, transfer of vGPCR/LacZ ${ }^{+}$cells into $\mathrm{Rag}^{-/-}$mice treated with DOX led to angioproliferation and, with time, to development of tumors containing both vGPCR/LacZ ${ }^{+}$and vGPCR/LacZ ${ }^{-}$cells. Taken together, these results indicate that vGPCR triggers angioproliferation directly and suggest a novel role for this molecule in the pathogenesis of Kaposi sarcoma.
\end{abstract}

\section{Introduction}

Human herpesvirus 8 (HHV8, also known as Kaposi sarcoma herpesvirus) is a gammaherpesvirus homologous to human EBV, simian herpesvirus saimiri (HVS), and murine gammaherpesvirus 68, discovered in 1994 by Chang et al. during screening of Kaposi sarcoma (KS) lesions (1). Since then, a number of studies have supported a role for HHV8 in the development of KS $(2,3)$, a multifocal highly vascularized neoplasm characterized by the presence of spindle-shaped cells, angiogenesis, and inflammatory infiltrates (reviewed in ref. 4). The HHV8 genome contains close to 100 open reading frames (ORFs), some displaying homology to cellular oncogenes with transforming capacity in vitro $(5-8)$.

Our lab and others have shown that transgenic expression of viral G protein-coupled receptor (vGPCR), the chemokine receptor encoded by HHV8 ORF74, induces development of angioproliferative lesions that resemble those seen in KS (9-11). In our model, the lesions are observed in ears, limbs, and tail and progress from erythematous lesions to nodules and tumors within 6 months (9). The lesions are composed of large numbers of spindleshaped $\mathrm{CD}^{+} 4^{+}$cells, vascular channels, and inflammatory cells. Conditional expression of vGPCR triggers expression of several angiogenic factors in lesional areas, and its inactivation results in regression of the angioproliferative lesions (12).

Nonstandard abbreviations used: DOX, doxycycline; FDG, fluorescein di- $\beta$-Dgalactopyranoside; FISH, fluorescence in situ hybridization; HHV8, human herpesvirus 8; iORF74, inducible ORF74; KS, Kaposi sarcoma; ORF74, open reading frame 74; $\mathrm{PB}$, phosphate buffer; $\mathrm{PFA}$, paraformaldehyde; Q-PCR, quantitative real-time PCR rtTA, reverse tetracycline-controlled transactivator; SSC, standard saline citrate; TRE, tetracycline-responsive element; vGPCR, viral G protein-coupled receptor.

Conflict of interest: The authors have declared that no conflict of interest exists. Citation for this article: J. Clin. Invest. doi:10.1172/JCI26666.
The relevance of vGPCR to KS pathogenesis is unclear. vGPCR appears to be expressed predominately during the lytic phase of the viral cycle in vitro (13), and few cells express this molecule in human tumors (14). However, the obvious ability of this receptor to induce a disease in mice that closely resembles KS in vivo has led several groups to propose that vGPCR has an important role in the pathogenesis of KS (9-11). The fact that vGPCR expression triggers an angiogenic gene program has led to the hypothesis that vGPCR contributes to angioproliferation and tumorigenesis via paracrine mechanisms $(9,10,15-17)$. The relevance of this mechanism in cells undergoing lysis has been questioned because expression of most vGPCR target genes is drastically curtailed during the lytic cycle (18). Others, however, have speculated that dysregulation of the viral gene program may lead to nonlytic expression of vGPCR and favor development of KS $(5,10)$. Yet no direct evidence for nonlytic expression of vGPCR in humans has been reported to date.

To address the role of VGPCR in angioproliferation and tumorigenesis, we have now developed a method for conditional and simultaneous expression of vGPCR and $\beta$-gal (LacZ). We phenotyped the cells expressing vGPCR and LacZ within lesional areas, mapped their distribution in early lesions and tumors, and tested their relevance to angioproliferation and tumorigenesis. Here we show that the vGPCR/ LacZ cells express markers found in endothelial progenitor cells, that they proliferate upon transgene activation (doxycycline [DOX] treatment), and that they transfer disease to immunodeficient $R a g 1^{-/-}$mice. Finally, we show that vGPCR/LacZ ${ }^{+}$cells surround tumors but that they are scarce within them. These results point to an early autocrine component and suggest a novel tumorigenic mechanism.

\section{Results}

Generation of mice expressing $\nu$ GPCR and LacZ conditionally. In order to generate a molecular marker for cells expressing vGPCR in 

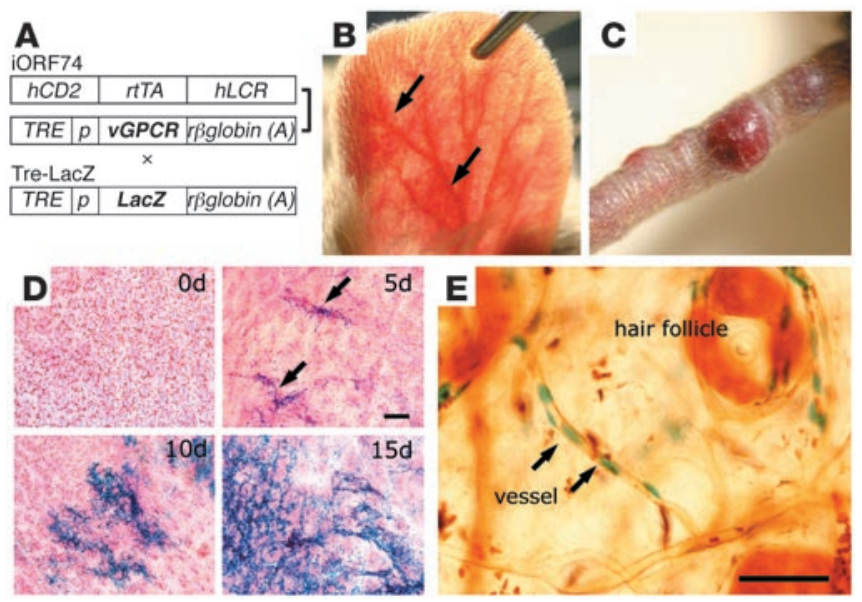

Figure 1

vGPCR/LacZ ${ }^{+}$cells accumulate over time in lesional areas. (A) iORF74 mice were generated by coinjection of a DOX-dependent activator and responder $V G P C R$ transgene (bracket indicates transgene cointegration). iORF74 mice and TRE-LacZ mice were crossed (X) to generate iORF74/LacZ mice. $h L C R$, locus control region; $p, C M V$ minimal promoter; $r \beta$ globin $A$, rabbit $\beta$ globin polyadenylation signal. (B and $\mathbf{C})$ iORF74/LacZ mice develop highly vascularized lesions in the ears (B, arrows) and tumors in the tail (C), depending on the length of DOX treatment (30 days and 100 days respectively). (D) $\beta$-gal whole-mount histochemistry of ears from iORF74/LacZ mice untreated (0 d) or treated with DOX for 5,10 , and 15 days ( $n=6$ ears per time point). No blue cells were found in ears of control mice (not shown) or untreated iORF74 mice. After 5 days of DOX treatment, small clusters of blue cells were detected (arrows). The number and the size of clusters increased over time. Scale bar: $100 \mu \mathrm{m}$. (E) Visualization of the vascular tree and vGPCR/LacZ $Z^{+}$cells using biotinylated $L$. esculentum lectin and $\beta$-gal whole-mount histochemistry. After 10 days of DOX treatment, vGPCR/ $\mathrm{LacZ}^{+}$cells were present in small capillaries or venules (arrows) and surrounding hair follicles. Scale bar: $20 \mu \mathrm{m}$.

vivo, we took advantage of the ability of reverse tetracycline-controlled transactivator ( $\mathrm{rtTA}$ ) of the Tet-On system (Clontech) to bind to and activate multiple tetracycline-responsive promoters. We have previously described the generation of transgenic mice expressing vGPCR conditionally (12). In these mice, referred to as inducible ORF74 (iORF74) mice, expression of the rtTA activator is controlled by a segment of the human $C D 2$ promoter ( $b C D 2-r t T A$ ), and $v$ GPCR expression is controlled by the tetracycline-responsive element (TRE-vGPCR) (Figure 1A). To facilitate the identification and characterization of the cells expressing vGPCR, we generated new transgenic lines carrying a TRE-LacZ transgene (Figure 1A). Five lines of TRE-LacZ mice were crossed to iORF74 mice to select lines responding to the activator transgene. LacZ expression was silent in most tissues in the absence of the activator transgene (Supplemental Table 1; supplemental material available online with this article; doi:10.1172/ JCI26666S1). Two lines carrying all 3 transgenes showed LacZ expression in the ears after DOX treatment (not shown). Coexpression of $L a c Z$ and $v G P C R$ was confirmed by quantitative realtime PCR (Q-PCR) on sorted $\mathrm{LacZ}^{+}$cells obtained from the ears (12). Treatment of the iORF74/LacZ mice with DOX for 40-60 days led to development of highly vascularized angiogenic lesions in the ears (Figure 1B). Longer treatment (over 100 days of DOX treatment) led to development of nodules and tumors in the tail (Figure 1C) and paws. Histological analysis of these lesions showed intense angioproliferation and accumulation of inflammatory cells (not shown).

$v G P C R / L a c Z^{+}$cells are present in areas where angioproliferative disease is observed. To study the distribution of vGPCR/LacZ $\mathrm{L}^{+}$cells in iORF74/LacZ mice, we collected several tissues before and after DOX treatment. Thymus, spleen, lymph nodes, ear, paw, skeletal muscle, liver, kidney, and other organs summarized in Supplemental Table 1 were processed for histology and $\beta$-gal histochemistry. As shown in Supplemental Table 1, untreated iORF74/LacZ mice showed few if any LacZ-positive cells in the tissues analyzed. In contrast, LacZ $Z^{+}$cells were clearly present in skin and thymus but not in spleen or LN (Supplemental Figure 1). vGPCR/LacZ+ cells were also detected with lower frequency in skeletal muscle, testis, and intestine. No vGPCR/LacZ ${ }^{+}$cells were detected in any of the other organs examined, including heart, liver, and kidney (Supplemental Figure 1). Histological analysis of the areas in which vGPCR/LacZ ${ }^{+}$cells were present showed angioproliferative lesions with varying degrees of severity. The most pronounced lesions were observed in skin of the ears, paws, and tail, areas where the largest number of $\mathrm{vGPCR} / \mathrm{LacZ}^{+}$cells were observed. The only exception was the thymus, where, despite a large number of vGPCR-expressing cells, no lesions were detected.

$v G P C R / L a c Z^{+}$cells accumulate in the ears as a function of DOX treatment. vGPCR expression in the ears of iORF74 mice increases with the duration of DOX treatment (12), which suggests that either vGPCR is expressed at higher levels by a fixed number of cells or that an increased number of cells accumulate in the lesional areas over time. To discriminate between these possibilities, we examined the distribution of $\mathrm{vGPCR} / \mathrm{LacZ}^{+}$cells in whole-mount preparations of ears collected at various time points. Ears from iORF74/LacZ mice ( $n=6$ per time point) were collected before (day 0) or after DOX treatment (days 1, 3, 5, 10, and 15). Untreated (iORF74/LacZ) littermates were used as controls. Before DOX treatment, no vGPCR/LacZ ${ }^{+}$cells were detected in ears of iORF74/LacZ mice or in controls. After 1 or 3 days of DOX treatment, fewer than $10 \mathrm{vGPCR} / \mathrm{LacZ}^{+}$cells were detected per ear (Figure 1D and data not shown). By day 5 , small aggregates of LacZ--expressing cells were present in the middle area of the ears, forming small clusters (Figure 1D). By day 10 , there was an increase in both the number of clusters and the number of vGPCR/LacZ ${ }^{+}$cells per cluster. The clusters were located throughout the whole ear, and their number continued to increase after 15 days of DOX treatment (Figure 1D). To investigate the location of the vGPCR/LacZ+ cells, we imaged the vascular tree using biotinylated Lycopersicon esculentum lectin. Interestingly, the majority of $\mathrm{vGPCR} / \mathrm{LacZ}^{+}$cells lined the surface or were closely associated with small capillaries or venules (Figure 1E). Very few vGPCR/LacZ+ cells were observed surrounding big vessels or in the ear parenchyma. These results indicate that an increase with time in the number of cells, rather than an increase in the expression of ORF74 per cell, accounted for the increased expression of VGPCR.

$v G P C R / L a c Z^{+}$cells express endothelial and progenitor markers. The coexpression of vGPCR and LacZ allowed us to examine the phenotype of cells expressing vGPCR. We used fluorescein di- $\beta$ D-galactopyranoside (FDG), a $\beta$-gal substrate, to detect LacZ ${ }^{+}$ cells by flow cytometry. After 80 days of DOX treatment, we observed a significant increase in the relative number of vascular cells $\left(\mathrm{CD} 31^{+}\right)$in ears of iORF74/LacZ mice compared with 

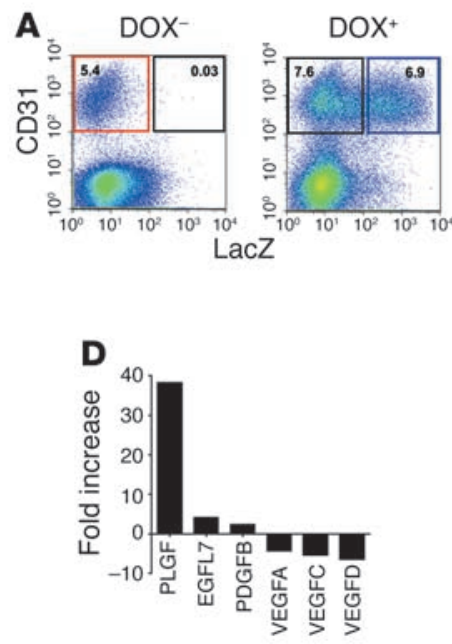

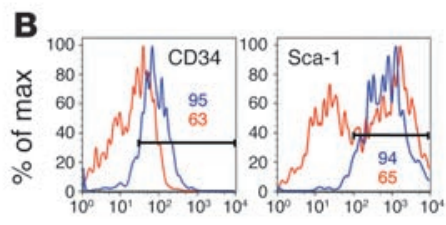

$\mathbf{E}$

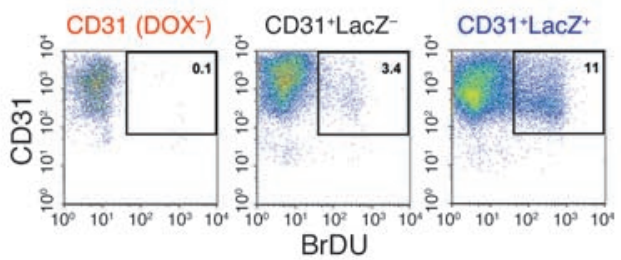

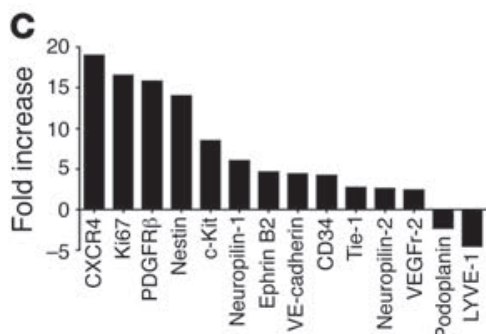

$\mathbf{F}$

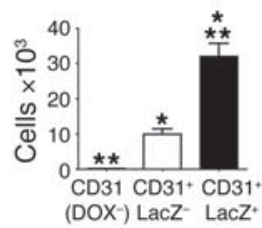

Figure 2

VGPCR/LacZ ${ }^{+}$cells express endothelial cell markers, angiogenic factors, and receptors. (A) Flow cytometric analysis of ear cells from untreated iORF74/LacZ mice (left dot plot) and iORF74/LacZ mice treated for 80 days with DOX (right dot plot). Approximately 50\% of CD31+ cells were vGPCR/LacZ+ (blue square). (B) Histogram representation of gated CD31+/LacZ+ ear cells of DOX-treated (blue lines) or CD31+ from untreated iORF74/LacZ control mice (red lines). Higher relative numbers of Sca-1- and CD34-positive cells were detected in CD31+/LacZ ${ }^{+}$cells compared with CD31+ cells from controls. Data are representative of 3 experiments $(n=12)$. Max, maximum. (C and D) Fold induction/reduction of mRNA expression of vascular cell markers $(\mathbf{C})$ or angiogenic secreted factors (D) in sorted CD31+/LacZ ${ }^{+}$cells compared with sorted CD31+ cells isolated from controls. CD31+/LacZ ${ }^{+}$cells were obtained from mice treated with DOX for 60 days. Data were normalized to ubiquitin, and the results are representative of 2 experiments. (E) BrdU incorporation in CD31 cells from iORF74/LacZ mice and controls. CD31+LacZ+ cells contained a higher relative number of $\mathrm{BrdU}^{+}$cells than the $\mathrm{CD} 31+\mathrm{LacZ}-$ cells (11\% versus $\left.3.4 \%\right)$. (F) Absolute number of proliferating CD31+ cells per ear in control and iORF74/LacZ-treated mice. In the control mouse group, a negligible number of CD31+ cells incorporated BrdU. Data are representative of 3 experiments, and values shown are the mean \pm SD. ${ }^{\star} P<0.01 ;{ }^{*} P<0.001$.

untreated mice $(P<0.01 ; n=15)$. Nearly half of the $\mathrm{CD} 31^{+}$cells expressed vGPCR/LacZ (Figure 2A). In contrast to the normal vasculature found in untreated iORF74/LacZ mice (Figure 2A), the majority of $\mathrm{CD} 1^{+} / \mathrm{LacZ}^{+}$cells in DOX-treated mice expressed the hematopoietic stem cell marker Sca-1 (Figure 2B). The majority $(96 \%)$ of the CD $31^{+}$vGPCR/LacZ ${ }^{+}$cells also expressed CD34. To extend these observations, we performed Q-PCR, focusing our analysis on markers for which antibodies are not readily available. When compared with control CD31 ${ }^{+}$ cells, the CD $31^{+}$vGPCR/LacZ ${ }^{+}$cells expressed higher levels of a number of endothelial and progenitor markers. These markers included VE-cadherin, ephrin B2, CD34, Tie-1, VEGFR-2, neuropilin1, and neuropilin2 (Figure $2 \mathrm{C}$ ). In addition, mRNA for molecules expressed by progenitor cells, such as CXCR4, nestin, $c$-Kit, and neuropilins, were also upregulated in vGPCR $/ \mathrm{LacZ}^{+}$cells. Likewise, expression of genes encoding secreted factors involved in angiogenesis and endothelial cell migration, i.e., placental growth factor ( $P l G F), P D G F \beta$, and EGFL7, was also upregulated (Figure 2D). In contrast, the expression of members of the VEGF family, such as $V E G F A, V E G F C$, and $V E G F D$, was downregulated, as was expression of the lymphatic markers LYVE-1 and podoplanin. Furthermore, there were no changes in the expression levels of the lymphatic marker VEGFR-3 (not shown). With the exception of increased expression of $P D G F R \beta$, there was no difference in the expression of pericyte markers (SMA, NG2, caldesmon, calponin, and RGS-5) between $\mathrm{vGPCR} / \mathrm{LacZ}^{+} \mathrm{CD} 31^{+}$and WT $\mathrm{CD} 31^{+}$cells (not shown). Together, these findings indicate that vGPCR/LacZ $Z^{+}$cells present in the angiogenic lesions express primarily endothelial cell markers and suggest that they derive from proliferation or recruitment of progenitor cells. $v G P C R / L a c Z^{+}$cells proliferate in situ. Next, we investigated whether the progressive accumulation of $\mathrm{vGPCR} / \mathrm{LacZ}^{+}$cells was due to in situ cell proliferation of cells in the lesional areas. We investigated the proliferation of the endothelial cells by BrdU incorporation. The iORF74/LacZ mice treated with DOX for 80 days and control iORF74/LacZ mice were injected with $2 \mathrm{mg}$ of BrdU 24 and 48 hours prior to sorting of $\mathrm{CD} 31^{+} \mathrm{LacZ}^{+}$and $\mathrm{CD} 1^{+} \mathrm{LacZ}^{-}$ear cells from DOX-treated mice and $\mathrm{CD} 31^{+}$ear cells from untreated controls. As shown in Figure 2E, both CD31+ $/$ $\mathrm{LacZ}^{+}$and $\mathrm{CD}^{3} 1^{+} / \mathrm{LacZ}^{-}$cells from DOX-treated iORF74/LacZ mice proliferated more ( $11 \%$ and $3.4 \%$, respectively) than $C D 31^{+}$ cells from control mice. Of note, a significantly higher relative and absolute number of proliferating cells $\left(\mathrm{BrdU}^{+}\right)$were found among the $\mathrm{CD} 1^{+} / \mathrm{LacZ}^{+}$cells than in the $\mathrm{CD} 31^{+} / \mathrm{LacZ}^{-}$cells (Figure $2 \mathrm{~F}$ ). These results indicate that $\mathrm{vGPCR} / \mathrm{LacZ}^{+}$cells were actively proliferating in situ.

$v G P C R / L a c Z^{+}$cells derive from cells present in the periphery. The accumulation of vGPCR/LacZ $Z^{+}$cells in lesional areas could be due to proliferation of precursors present in the tissue, derived from precursors present in circulation, or both. To address this issue, we first determined whether vGPCR/LacZ ${ }^{+}$cells were present in blood of DOX-treated mice. We did not detect such cells in circulation by flow cytometry or Q-PCR (not shown). However, low expression of vGPCR was observed in the BM of iORF74/LacZ mice, and it is possible that the methods we used to detect them in circulation may not have been sensitive enough.

To further determine whether BM-derived endothelial cell precursors contributed to the nascent vasculature, we transferred BM cells from GFP transgenic mice to lethally irradiated nontreated iORF74 mice. Four weeks afterwards, transplantation engraftment 
A

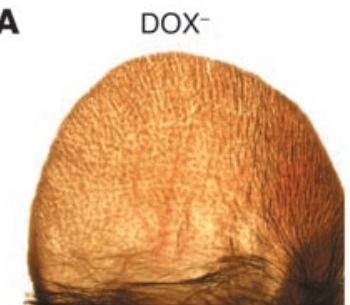

B

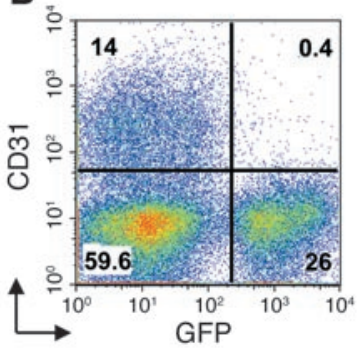

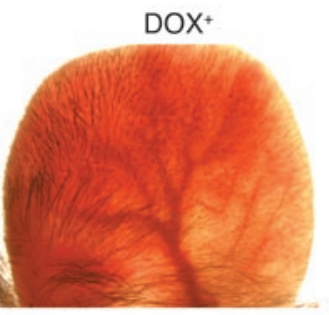

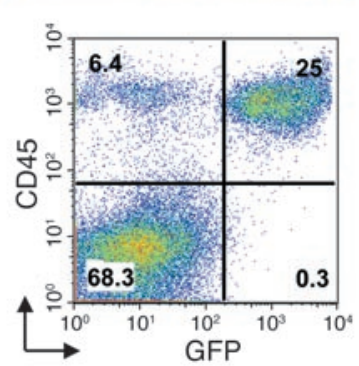

was confirmed by FACS analysis (70-85\% of the circulating cells were $\left.\mathrm{GFP}^{+}\right)$. The iORF74 mice, which contained a vGPCR-deficient, $\mathrm{GFP}^{+}$hematopoietic compartment, were fed normal $(n=10)$ or DOX-containing chow $(n=10)$. Animals on the control diet did not develop lesions in the ears (Figure 3A), but those treated with DOX for 14 weeks had significant inflammation and angioproliferation (Figure 3A). These results indicated that cells causing the angioproliferative disease were radioinsensitive but did not rule out that they derived from the bone marrow.

To investigate whether the bone marrow cells contributed to the generation of proliferating endothelial cells, we performed flow cytometric analysis. Ear cells were collected, processed, and stained with anti-CD31 and anti-CD45 Abs. The majority of endothelial cells $\left(\mathrm{CD} 31^{+}\right)$in iORF74 mice treated with DOX $(n=3)$ were GFP negative (Figure 3B), indicating that the newly formed vessels were not composed of $\mathrm{BM}$-derived $\mathrm{GFP}^{+}$cells. On the contrary, the majority of $\mathrm{CD} 45^{+}$cells in the lesions were also $\mathrm{GFP}^{+}$(Figure 3B), suggesting that hematopoietic cells accumulated in the angiogenic lesions.

The results discussed above suggested that the precursors could be present in the periphery, rather than the BM. To test this hypothesis, we examined the presence of precursor cells in ears of untreated iORF74/LacZ mice. To obtain a preparation with a minimal number of circulating cells, we perfused animals through

\section{Figure 4}

vGPCR/LacZ+ cells originate from cells present in adult ears. Ear cells from perfused untreated iORF74/LacZ mice were cultured in Matrigel in the presence or absence of $10 \mu \mathrm{g} / \mathrm{ml}$ DOX. $\beta$-gal histochemistry and Q-PCR were performed before culture (day 0 ) and after 4 , 10 , and 15 days in culture. (A) By day, 10 small clusters of blue cells and structures that resembled vessels (arrows) were observed. (B) Immunohistochemistry analysis of the clusters present at day 10 after culture showed the presence of $\mathrm{CD} 31^{+}$cells forming vessel-like structures. (C) Quantification of $\mathrm{LacZ}^{+}$cells in cultures of ear cells revealed a significant increase in the number of $\mathrm{VGPCR} / \mathrm{LacZ}^{+}$cells over time. (D) Expression of $V G P C R$ increased over time in cultured cells in the presence of DOX (detected by Q-PCR). Data were normalized to ubiquitin expression levels and are representative of 3 experiments. Values shown are the mean $\pm S D$. Statistical significance was calculated comparing values with those of day $0 .{ }^{*} P<0.05$. Scale bars: $20 \mu \mathrm{m}$.

\section{Figure 3}

vGPCR/LacZ+ cells are radioinsensitive. Lethally irradiated iORF74 mice transplanted with bone marrow from GFP+ donor mice were given a DOX-containing diet or a regular diet ( $n=10$ per group). (A) Lethal irradiation did not affect the development of angiogenic lesions in the ear skin of iORF74 mice (14 weeks of DOX treatment, right panel, compared with untreated iORF74 transplanted animal, left panel). (B) Flow cytometric analysis of ear cells shown in the right panel in $\mathbf{A}$. The majority of the GFP ${ }^{+}$cells in the ears were CD45+. Only a fraction of the CD $31^{+}$cells $(\sim 2.8 \%)$ were GFP+, indicating that the bulk of the proliferating $\mathrm{CD} 31^{+}$cells derived from the host and not from the donor $\mathrm{GFP}+\mathrm{BM} . n=3$.

the left ventricle with saline solution. After digestion of the ears, isolated cells $\left(10^{6}\right)$ were placed over coverslips coated with Matrigel and cultured in DMEM with or without DOX. At intervals, cells were stained for $\beta$-gal histochemistry or harvested for RNA extraction. In the absence of DOX, vGPCR/Lac $Z^{+}$cells were not found in cultures. However, as shown in Figure 4A, vGPCR/LacZ ${ }^{+}$cells formed clusters and tubes after 10 days in culture with DOX. The morphology of these cells suggested an endothelial origin, and most of them expressed the endothelial marker CD31 (Figure 4B). Quantification of $\mathrm{LacZ}^{+}$cells in culture revealed that the number of $\mathrm{LacZ}^{+}$cells in culture increased significantly over time in the group treated with DOX (Figure 4C). Coincidentally, the relative expression of vGPCR mRNA also increased with time in the cultures after addition of DOX (Figure 4D). These findings indicate that at least a subset of cells contributing to the angioproliferative lesions were already present in the ears prior to DOX treatment and that they proliferated in response to vGPCR expression. Taken together, these results show that cells with the potential to express vGPCR were already present in the periphery (ears) and that disease progression did not depend on endothelial cell precursors derived from the bone marrow.

$\nu G P C R / L a c Z^{+}$cells transfer angioproliferative disease. To determine whether vGPCR/LacZ $Z^{+}$cells were sufficient for the development of angioproliferative disease, we transferred vGPCR/LacZ ${ }^{+}$cells to ears of Rag1-deficient mice. In 5 independent experiments $(n=18)$, $3 \times 10^{4}$ sorted vGPCR/LacZ ${ }^{+}$cells were injected s.c. into the middle region of the dorsum of the ear of Rag1-deficient mice. PBS was injected in the contralateral ear. One group of mice was fed a DOX-containing diet; the other group was fed a regular diet.
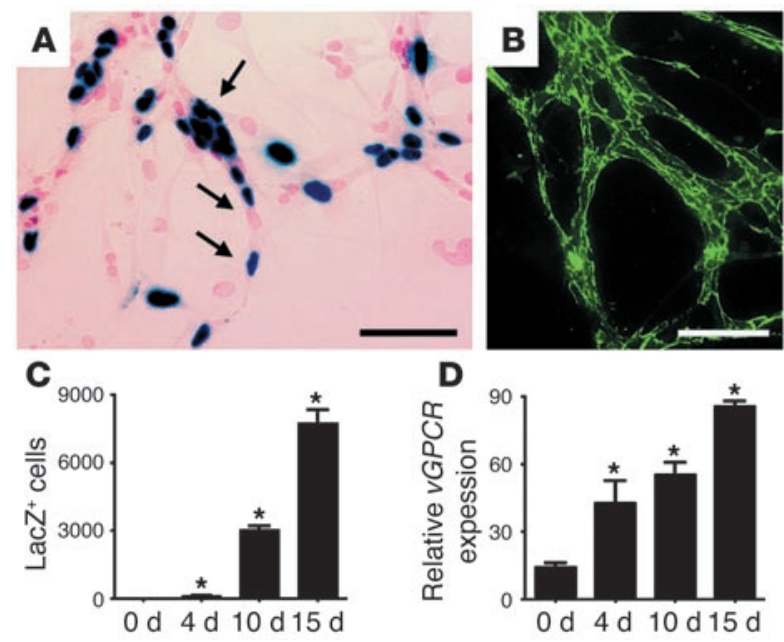
A

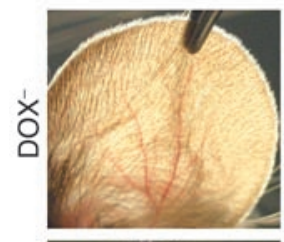

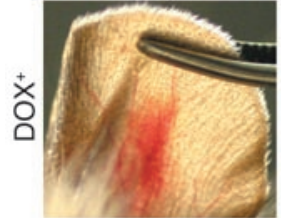

$50 d$

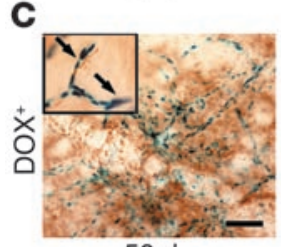

$50 \mathrm{~d}$

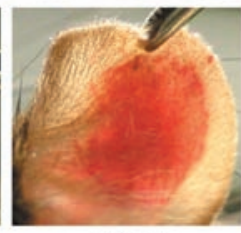

$120 \mathrm{~d}$

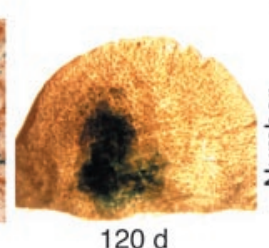

$120 \mathrm{~d}$

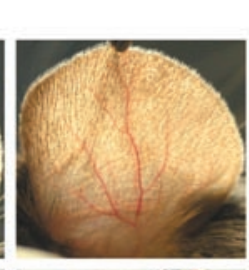

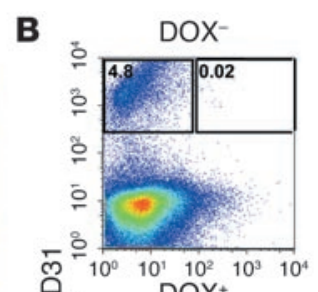

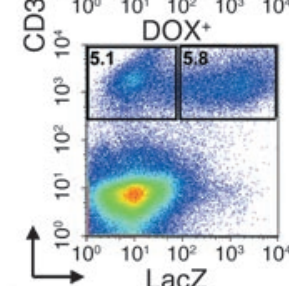

D

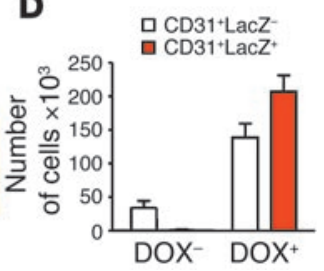

Ears were inspected weekly. Mild angiogenic activity was first detected in ears of mice injected with vGPCR/LacZ ${ }^{+}$cells $30-40$ days after DOX treatment was started. Larger angiogenic lesions were observed after 50 days, and by 120 days, they occupied more than $50 \%$ of the ear (Figure $5 \mathrm{~A}$ ). Neither the ears of the mice from the control group treated with regular chow (Figure 5A) nor the contralateral ears of DOX-treated mice injected with PBS had developed angiogenic lesions 120 days after transfer. By 240 days of treatment, mice transferred with vGPCR/LacZ ${ }^{+}$cells and treated with DOX had swollen ears and large erythematous lesions that occupied almost the whole ear (data not shown). Flow cytometric analysis of these samples $(n=2)$ showed that, among the total ear cells $\left(3.47 \pm 0.3 \times 10^{6}\right.$ cells $), 5.8 \%$ were $\mathrm{CD}^{2} 1^{+} \mathrm{LacZ}^{+}$ $\left(0.21 \times 10^{6}\right.$ cells) (Figure $\left.5 \mathrm{~B}\right)$. Thus, the number of CD31+ ${ }^{+} \mathrm{LacZ}^{+}$ cells isolated at this point was 7-fold higher than the number of vGPCR/LacZ ${ }^{+}$cells injected. We also detected an increase in total number of CD31+LacZ ${ }^{-}$cells in the same ears (3-fold) (Figure 5D). $\beta$-gal histochemistry of ear whole-mount preparations from transplanted mice treated for 50 or 120 days with DOX showed that $\mathrm{vGPCR} / \mathrm{LacZ}^{+}$cell aggregates were found in the same areas where angiogenic activity was observed (Figure 5C). In these areas, we found many vGPCR/LacZ ${ }^{+}$cells within or near sprouting vessels (Figure 5C). Taken together, these results indicate that transfer of vGPCR/LacZ ${ }^{+}$cells to ears of DOX-treated Rag1-deficient mice leads to intense angioproliferation.

\section{Figure 6}

Tumors from DOX-treated iOFR74/LacZ mice contain few LacZ+ cells. (A) Representative picture of a tail tumor from an iORF74/LacZ mouse treated with DOX for 200 days. Arrowheads indicate areas of sectioning. (B) $\beta$-gal histochemistry of a transversal section from the region indicated by the red arrowhead. Very few vGPCR/LacZ+ cells were present within the tumor (red dotted line and arrowhead). (C) Areas flanking the tumor (black arrowhead) showed the presence of a large number of vGPCR/LacZ+ cells. (D) Higher magnification of the area in $\mathbf{C}$ showing the presence of a high number of blue cells associated with vessels and an incipient tumor (indicated by the red dotted line). Results are representative of 6 different tumors. Scale bars: $100 \mu \mathrm{m}$.

\section{Figure 5}

vGPCR/LacZ+ ear cells transfer angioproliferative disease to Rag $1^{-/-}$ mice. $(A)$ Sorted vGPCR/LacZ ${ }^{+}$cells $\left(3 \times 10^{4}\right)$ were injected s.c. into the middle region of the dorsum of the ear of Rag1-deficient mice. Angiogenic lesions were visible in the ears of the group treated with DOX (lower left panel) but not in the ears of untreated animals (upper panels). (B) Flow cytometric analysis of ears that received vGPCR/ LacZ ${ }^{+}$cells. CD31+ LacZ+ cells were absent in mice treated with regular chow (upper dot plot) but present in mice treated with DOX (lower dot plot). (C) Representative $\beta$-gal histochemistry of whole-mount preparation shows the high coincidence of $\mathrm{VGPCR} / \mathrm{LacZ}^{+}$cell aggregates (blue) with areas with angiogenic activity (shown in A). Vessel sprouting was occasionally observed (arrows, insert in C). (D) After 120 days of DOX treatment, the number of CD31+LacZ+ cells was 7-fold higher than the number of cells injected. Values shown are the mean $\pm S D$. Scale bar: $50 \mu \mathrm{m}$.

Few $v$ GPCR $/ \mathrm{LacZ}^{+}$cells are present within tumors. At an early stage, KS is characterized by intense angioproliferation with the formation of dilated vascular spaces in the epidermis, interstitial inflammatory cells, and extravasated red cells. Later, nodular tumors composed of spindle-shaped cells were observed. To further study the contribution of vGPCR/LacZ ${ }^{+}$cells to tumorigenesis, we treated iORF74/ LacZ mice with DOX until tumors were established on tails (usually after 150 days of DOX treatment) and analyzed the distribution of the vGPCR/LacZ ${ }^{+}$cells within these tumors. We stained sections obtained from tumors or adjacent areas (Figure 6A). We found a large number of $\mathrm{vGPCR} / \mathrm{LacZ}^{+}$cells in sections adjoining the tumors (Figure 6, C and D). Strikingly, very few vGPCR/ $\mathrm{LacZ}^{+}$cells were present within tumors (Figure 6B). These results confirm the previous observations that few vGPCR ${ }^{+}$cells are present within tumors of transgenic mice (9).

$\nu \mathrm{GPCR} / \mathrm{LacZ}^{+}$cells are critical for the development of tumors. To investigate whether the transfer of vGPCR/Lac $Z^{+}$cells would lead to development of tumors, we transferred sorted vGPCR/ LacZ $^{+}$cells $\left(3 \times 10^{4}\right)$ from ears of DOX-treated iORF74/LacZ mice to tails of Rag $1^{-/-}$mice. After 200 days of DOX treatment, tumors were present in areas where the vGPCR/LacZ ${ }^{+}$cells were injected $(n=8)$ (Figure 7A). Immunohistochemical analysis revealed that the majority of cells within the tail tumors were CD $31^{+}$cells (Figure 7B). Strikingly, and in great contrast with the observation that many vGPCR/LacZ ${ }^{+}$cells were present dur-
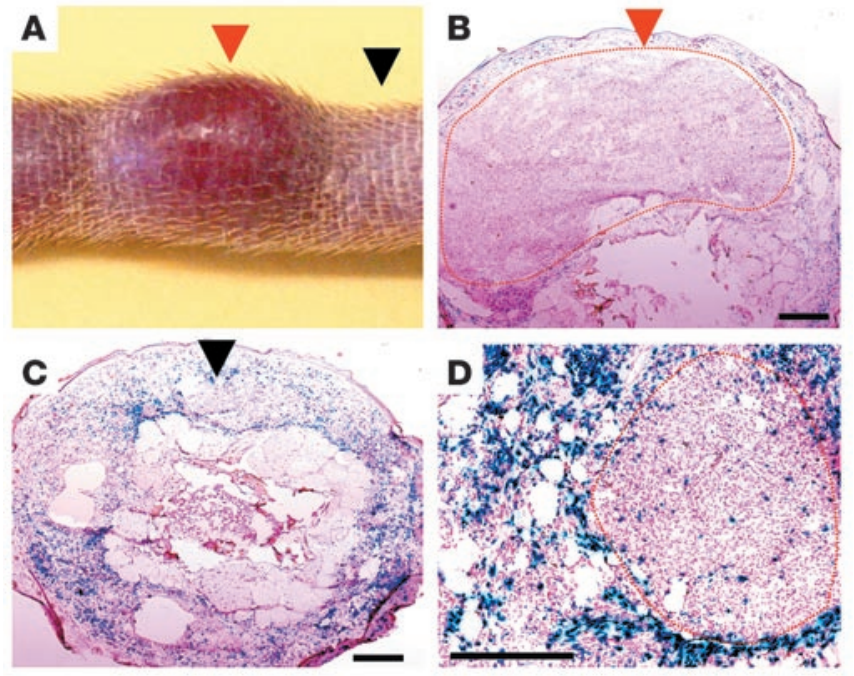

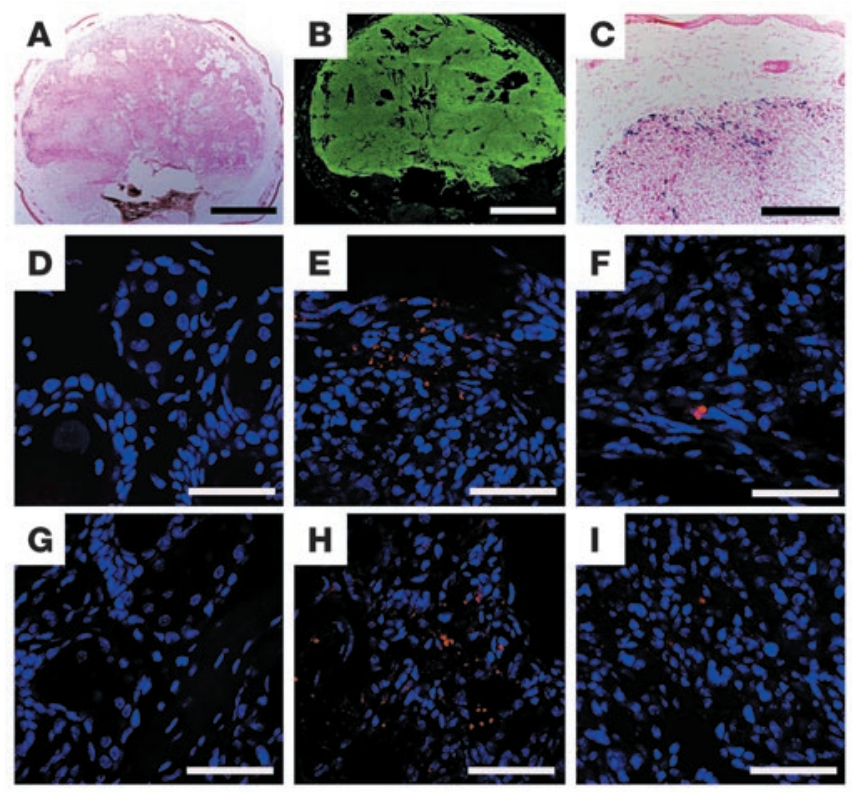

ing early stages of angioproliferation (shown in Figure 5C), very few LacZ-positive cells were found within the tail tumors in Rag1 $1^{-1-}$ recipient mice (Figure $7 \mathrm{~B}$ ). vGPCR/LacZ ${ }^{+}$cells accumulated in the periphery of the tumors (Figure $7 C$ ) in an arrangement similar to that observed in the iORF74/LacZ mice treated for a long time with DOX (Figure 6).

To investigate whether the reduced expression of LacZ was due to reduced transcription and whether $v$ GPCR expression was also reduced, we performed fluorescence in situ hybridization (FISH). Tail tumors of $\operatorname{Rag} 1^{-/-}$recipient mice $(n=2)$ were tested using specific probes designed for both transgenes ( $L a c Z$ and $v$ GPCR). Tail sections of iORF74 mice treated with DOX for 60 days and WT mice were used as positive and negative controls. No signal was detected for $\nu$ GPCR or LacZ in WT mice (Figure 7, D and G). As expected, mRNA for $L a c Z$ and $v$ GPCR (Figure 7, E and H, respectively) was detected in tail sections of iORF74 mice treated with DOX. However, as shown in Figure 7, sections from tumors resulting from transplanted $v G P C R / \mathrm{LacZ}^{+}$cells showed very low expression of mRNA for $L a c Z$ and $\nu$ GPCR (Figure 7, F and I, respectively). No signal was detected when scrambled probes were used in sections from controls or animals in the experimen-

\section{Figure 8}

LacZ- cells in tumors of transplanted Rag mice derive from LacZ+ cells. Sorted vGPCR/LacZ+ cells from the ears of DOX-treated iORF74/LacZ mice were transplanted into the tail skin of $R a g 1^{-/-}$mice. After transfer, mice were treated with DOX for 120 days. (A) An incipient tumor (nodule) in the area where vGPCR/LacZ+ cells were injected contained both $\mathrm{LacZ}^{+}$and LacZ ${ }^{-}$cells (red circles). (B) The nodule is mainly composed of $\mathrm{CD} 1^{+}$cells (green). (C and D) Representative $\mathrm{LacZ}^{+}$and $\mathrm{LacZ}^{-}$ cells before (C) and after (D) laser capture microdissection. (E) Relative levels of $V G P C R$, neomycin, and LacZ DNA in total genomic DNA from microdissected samples ( $n=5$ per group). (F) Normalized values of DNA content of $\mathrm{LacZ}^{+}$and $\mathrm{LacZ}^{-}$cells microdissected from the tail nodule in recipient mice (represented by the red circles in $\mathbf{A}$ ). LacZ ${ }^{+}$ and LacZ- samples collected within the tumor have a similar DNA profile, suggesting that they derive from the same source (vGPCR/LacZ+ cells). Scale bars: $50 \mu \mathrm{m}$ (A), $100 \mu \mathrm{m}$ (B).

\section{Figure 7}

Adoptive transfer of $\mathrm{vGPCR} / \mathrm{LacZ}^{+}$cells promotes development of tumors. Sorted vGPCR/LacZ+ cells $\left(3 \times 10^{4}\right)$ from the ears of DOXtreated iORF74/LacZ mice were transplanted into tail skin of Rag 1-/mice. Tumors in the tails of recipient mice were observed after 200 days of DOX treatment. (A) $\beta$-gal histochemistry of the tail tumor. (B) Tail tumors were mainly composed of CD31+ cells. (C) Higher magnification of the tail tumor seen in $\mathbf{A}$ shows the presence of both $\mathrm{LacZ}^{+}$ and LacZ- cells. (D-I) Quantum dot FISH of sections of WT, iORF74/ LacZ, and Rag $1^{-/-}$recipient mice. (D and G) FISH in tail sections of WT mice for LacZ (D) and $v G P C R(\mathbf{G})$ probes, respectively. (E and $\mathbf{H})$ FISH in adjacent tail sections of iORF74/LacZ mice treated for 60 days with DOX, LacZ (E), and $v G P C R(\mathbf{H})$ probes, respectively. (F and I) FISH in adjacent tail sections of Rag ${ }^{-1-}$ recipient mice treated for 200 days with DOX, LacZ (F), and $v G P C R$ (I) probes, respectively. Scale bars: $400 \mu \mathrm{m}$ (A and B); $100 \mu \mathrm{m}$ (C); $40 \mu \mathrm{m}$ (D-I).

tal group (data not shown). These results show that mRNA for LacZ and $v$ GPCR was not expressed by many cells within tumors of $R a g 1^{-1-}$ recipient mice treated with DOX.

$v$ GPCR $/ \mathrm{LacZ}^{+}$cells are the likely source of many $v \mathrm{GPCR} / \mathrm{LacZ} \mathrm{Z}^{-}$cells within transplanted tumors. The low frequency of vGPCR/LacZ $Z^{+}$cells within the tumors raised an important question: Do the LacZ- cells within the tumor derive from the transplanted cells, or do they represent host cells that may have proliferated? To discriminate between these 2 possibilities, we performed laser capture microdissection of cells present in tumors resulting from transplant of vGPCR/LacZ $Z^{+}$cells into the tails of $\operatorname{Rag} 1^{-/-}$mice $(n=5)$. Small tumors (nodules) were present within the injected area 120 days after transplantation and DOX treatment (Figure 8A). LacZ- and $\mathrm{LacZ}^{+}$ cells were readily seen within these incipient tumors (Figure $8 \mathrm{~A}$ ), which had a high density of $\mathrm{CD} 31^{+}$cells (Figure $8 \mathrm{~B}$ ). Laser microdissected $\mathrm{LacZ}^{+}$and $\mathrm{LacZ}^{-}$cells from the nodule (represented in Figure 8, C and D, before and after microdissection, respectively) were analyzed for DNA profile comparison. Samples obtained from the vicinity of the nodules (Rag $1^{-/}$host tissue) and from iORF74/LacZ (treated for 60 days with DOX) and WT mouse tails were used as controls. Genomic DNA was extracted from these
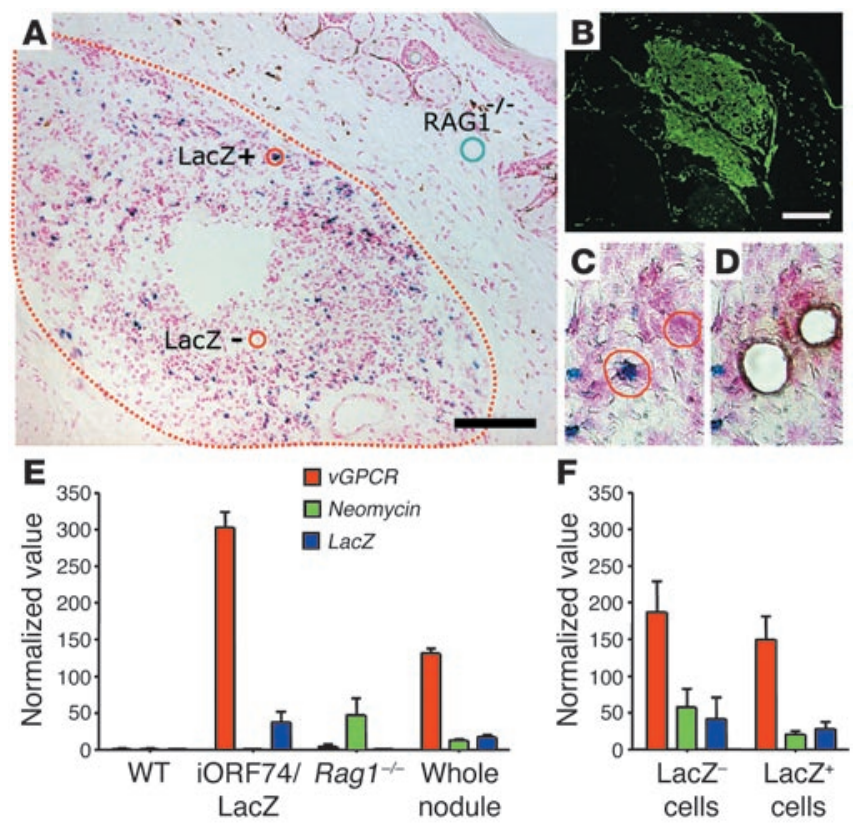
samples, and Q-PCR was used to analyze the relative amounts of $v$ GPCR, neomycin resistance, and LacZ DNA in 7 independent experiments. As expected, samples obtained from microdissected iORF74/LacZ tails contained $v$ GPCR and LacZ DNA (Figure 8E), and samples from (WT) controls had no $v$ GPCR or LacZ DNA. Cells from nonlesional areas of $\operatorname{Rag} 1^{-/-}$recipients were positive for the neomycin-resistance gene, as expected, since this gene is present in the targeting cassette used to disrupt the Rag1 gene (Figure 8E). Interestingly, the DNA profiles of $\mathrm{LacZ}^{+}$and $\mathrm{LacZ}^{-}$ cells within the nodules were very similar (Figure 8F) and matched the DNA profile found in whole microdissected nodules present in $R a g 1^{-/-}$recipient mice and the profile of samples obtained from iORF74/LacZ tails (Figure 8E). Taken together, the results suggest that many LacZ $Z^{-}$cells present within the nodules in Rag $^{1^{-/-}}$recipient mice derive from $v G P C R / \mathrm{LacZ}^{+}$cells.

\section{Discussion}

Several studies implicate the chemokine receptor vGPCR in the pathogenesis of KS (19-21). Expression of this molecule in transgenic mice leads to development of a disease that resembles KS both clinically and histologically (9-12). vGPCR-expressing cells are found within the lesions and appear to produce many paracrine factors that affect angiogenesis and inflammation (12). In the present study, we explored mechanisms whereby vGPCR causes disease. We report that the majority of vGPCR/LacZ ${ }^{+}$cells present in the lesions express endothelial cell markers, proliferate, secrete angiogenic factors, and cause angioproliferative disease when transferred into the skin of $\operatorname{Rag} 1^{-1-}$ recipient mice. These results question the prevailing paracrine model for neoplasia induced by vGPCR $(9,10,17)$ and shine new light on the role of this molecule in pathogenesis.

The main finding of this study is that vGPCR promotes angioproliferation directly when expressed in endothelial cells of transgenic mice. Thus far, it has been inferred that vGPCR induces angioproliferation and tumorigenesis through indirect (paracrine) mechanisms $(9,10,17)$. The use of a conditional system coupled to the use of a surrogate marker allowed us to analyze in detail the mechanism of angioproliferation and to demonstrate that vGPCRexpressing cells accumulated in lesional areas over time. Unexpectedly, we found that a sizeable portion of these cells expressed proliferation markers and incorporated BrdU. Moreover, we found that sorted vGPCR/LacZ ${ }^{+}$cells stimulated with DOX proliferated both in vitro on Matrigel and in vivo when transferred to Rag $1^{-/-}$ recipient ears. These findings indicate that VGPCR induces angioproliferation directly and suggest that autocrine mechanisms are important in the context of this transgenic model.

The phenotype and the origin of the cell driving angioproliferation in KS remain unclear. The most common cell type found within KS tumors is the spindle cell. While some studies suggest that spindle cells have an endothelial origin $(8,15)$, others have suggested that they derive from mesenchymal cells (22) or lymphatic endothelial cells (23). It has also been suggested that HHV8 may infect an endothelial cell precursor population in vivo, but evidence for this is lacking (24). Our studies support the hypothesis that vGPCR is expressed by an endothelial progenitor in transgenic models (12). The vGPCR/LacZ ${ }^{+}$cells express CD34, CD31, VEGFR-2, and nestin, markers associated with endothelial lineage (25-30). They also express molecules involved in angiogenesis and cell proliferation (PlGF, PDGF, and EGFL7) $(31,32)$. It is unclear at this point whether the expression of the markers is restricted to a few
vGPCR/LacZ ${ }^{+}$cells or whether these molecules are expressed by all cells. Finally, Wang et al. (24) have reported that HHV8 infection induces lymphatic transdifferentiation of human endothelial cells. Our studies indicate that continued expression of vGPCR (for at least 2 months) does not induce expression of lymphatic markers, such as podoplanin, LYVE-1, and VEGFR-3, in vGPCR/LacZ ${ }^{+}$cells, suggesting that either other viral genes are responsible for lymphatic endothelial differentiation or that additional changes are required for VGPCR to induce this program.

Compared with the control CD $31^{+}$endothelial cells, the vGPCR/ $\mathrm{LacZ}^{+}$cells overexpressed mRNA for progenitor or stem cell markers (CD34, c-Kit, Sca-1, CXCR4, and nestin). A natural source for such cells would be the BM, as there is evidence supporting the existence of BM-derived stem or endothelial progenitor cells in adults (33-35). Furthermore, vGPCR is expressed at very low levels in the $\mathrm{BM}$ of DOX-treated iORF74/LacZ mice. However, the BM transfer experiments showed that depletion of the radiosensitive hematopoietic compartment did not ablate disease and that the majority of $\mathrm{CD} 31^{+}$cells recovered from the ears were from the recipient and not the donor $\left(\mathrm{GFP}^{+}\right)$. These findings do not rule out the possibility that the precursors for the vGPCR/LacZ ${ }^{+}$cells found in the periphery may ultimately originate from the $\mathrm{BM}$ but indicate that $\mathrm{BM}$-derived endothelial cells do not contribute significantly to ongoing angioproliferative disease in adult iORF74/LacZ mice.

Our results strongly suggest that vGPCR/LacZ $Z^{+}$cells derive from progenitors present in the periphery. We suspect that these cells are not unique to the ear. Our working hypothesis is that the $b C D 2$ promoter fortuitously targets an endothelial precursor cell (most likely to be found associated with endothelium) that proliferates and expresses a number of angiogenic genes as a direct consequence of vGPCR expression. More studies are clearly required to trace the origin of the progenitor cells found in the periphery.

Our results indicate that vGPCR induces development of a 2stage disease. The first phase is characterized by angioproliferation and inflammation, and the second phase is characterized by tumorigenesis. As shown here, vGPCR has a direct role in angioproliferation. The failure to detect a direct role for vGPCR in angioproliferation in vivo in previous studies was probably caused by the lack of an appropriate animal model. It was difficult to predict where the tumors would form. In addition, it has been technically difficult to isolate vGPCR ${ }^{+}$cells from tumors and study their properties in vitro. We postulate that the vGPCR-driven autocrine program leads to intense proliferation of endothelial progenitors and accumulation of many $\mathrm{CD} 31^{+}$cells. There are 2 lines of evidence for autocrine mechanisms in the process. First, vGPCR function in vivo depends on its ability to signal constitutively $(36,37)$. Second, vGPCR/LacZ ${ }^{+}$cells express receptors for the angiogenic and inflammatory factors they produce (ref. 12 and data not shown). Additional paracrine mechanisms cannot be ruled out, as there is a considerable inflammatory response in the lesions. These inflammatory cells may contribute to disease development by producing factors that may have multiple functions, including the regulation of vGPCR activity. In fact, the ability of vGPCR to cause disease is also dependent on its ability to respond to chemokines (36).

The second phase of the disease is characterized by tumor development. Interestingly, vGPCR/LacZ ${ }^{+}$cells, which were numerous during the first phase, were scarce within the tumors during the second phase. The low frequency of $\mathrm{vGPCR}^{+}$cells in transgenic tumors is in agreement with original observations from our lab and others $(9-11,36)$. The presence of few cells expressing vGPCR 
within the tumors and the ability of vGPCR to induce expression of a large number of paracrine factors led to the concept that vGPCR may induce tumorigenesis via paracrine mechanisms (9-11, 36). Results presented here suggest that the tumor cells may derive from cells that earlier expressed vGPCR. It is unclear at this time what process would favor inactivation of vGPCR expression in these cells. One possibility would be that expression of vGPCR in a progenitor population would induce its differentiation into cells that no longer express the transgene. However, terminal differentiation of progenitor or precursor cells is unlikely to result in formation of the tumors unless vGPCR contributes directly to their transformation. A number of studies support a role for vGPCR in transformation. Transfection of vGPCR into a 3 T3 cell line induces proliferation and transformation (38), and expression of vGPCR in HUVECs leads to immortalization (21). Furthermore, molecules downstream of vGPCR, such as Akt and GTPase Rac1, can transform cells $(37,39)$. Thus, dysregulated expression of vGPCR could directly contribute to tumorigenesis in our model. This hypothesis would be alternative to the paracrine hypothesis for tumorigenesis raised by several groups, including ours (9-11, 36). We should note, however, that while our results strongly suggest that the tumors derive from vGPCR-expressing cells, only lineage-mapping experiments can conclusively rule out the possibility that paracrine mechanisms play a role in tumorigenesis in this transgenic model.

The present results raise new questions about the ultimate role of vGPCR during the viral infection. As briefly discussed in the introduction, vGPCR is a gene expressed during the lytic phase of the viral infection in vitro (13). Thus, the natural course for virally infected cells would be death, not proliferation. Results shown here and elsewhere conclusively demonstrate that vGPCR can directly trigger proliferation of fibroblasts and endothelial cells in vitro $(21,38)$ and in vivo (this work). So, how to reconcile these findings? One possibility is that dysregulated expression of vGPCR during the latent phase (or during aborted lytic cycles) of HHV8 infection in humans may trigger the initial phases of the disease $(5,40)$. This model is attractive, but there is no evidence to date to support it in the context of HHV8 infection. Formal proof of a pathogenic role of vGPCR can only be provided by infection of animals with HHV8 and HHV8 mutants lacking vGPCR. Such experiments are not possible at present because there are no animal models for HHV8 infection. Development of such models will be required to determine whether vGPCR is a critical determinant of disease, to determine whether vGPCR expression can indeed be triggered outside the lytic phase in vivo, and to define which factors, if any, contribute to its dysregulated expression.

\section{Methods}

Mice. iORF74 mice and TRE-LacZ mice were generated as previously described (12). iORF74 mice were mated to TRE-LacZ mice, and double transgenic mice (iORF74/LacZ) were identified by PCR amplification of tail DNA. Rag1-deficient mice were purchased from Jackson Laboratory. Transgenic mice were kept under pathogen-free conditions. All experiments involving animals were performed with approval from the Institutional Animal Care and Use Committee of the Mount Sinai School of Medicine.

Histology and immunohistochemistry. For $\beta$-gal histochemistry, 8- $\mu \mathrm{m}-$ thick fresh-frozen sections were fixed in $2 \%$ paraformaldehyde (PFA) in PBS and incubated overnight in the presence of 5-bromo-4-chloro-3indolyl- $\beta$-D-galactopyranoside (X-gal; Roche Molecular Biochemicals), as previously described (41). Nuclear Fast Red (Vector Laboratories) was used for counterstaining. For immunohistochemical staining, freshfrozen sections were fixed with ice-cold acetone for 10 minutes, dried, and washed with PBS. Slides were incubated with purified anti-CD31 (BD Biosciences - Pharmingen) for 1 hour at room temperature, followed by incubation with Alexa Fluor 488 labeled goat anti-rat antibody (Invitrogen Corp.) for 30 minutes.

Whole-mount lectin and $\beta$-gal vessel staining. Whole-mount lectin and $\beta$-gal staining protocols were combined to visualize vGPCR/LacZ ${ }^{+}$-expressing cells and blood vessels in whole-mount preparations of ears as described $(12,42)$. Briefly, biotinylated L. esculentum lectin (100 $\mu \mathrm{g}$; Vector Laboratories) was injected i.v. into mice under deep anesthesia followed by fixation perfusion through the left ventricle. The ears were dissected and $\beta$-gal histochemistry was performed as described above; biotinylated lectin was visualized using VECTASTAIN ABC and DAB Peroxidase Substrate kits according to the manufacturer's instructions (Vector Laboratories). Finally, the tissues were dehydrated and mounted on slides. In some experiments, $\beta$-gal histochemistry was performed on the ear skin followed by counterstaining with Nuclear Fast Red (Vector Laboratories).

Flow cytometry and BrdU incorporation. Ears were collected and processed as described (12). To identify vGPCR/LacZ ${ }^{+}$cells, $5 \times 10^{6}$ cells were incubated with FDG (Invitrogen Corp.) according to the manufacturer's instructions. FDG-loaded cells were stained with the directly conjugated monoclonal antibodies against CD31 (MEC 13.3), CD34 (RAM34), Ly-6A/E (Sca-1), and CD45 (30-F11) (BD Biosciences - Pharmingen). For analysis of cell proliferation, iORF74/LacZ mice treated for 80 days with DOX food were injected i.p. with BrdU (2 mg; Sigma-Aldrich) 48 and 24 hours before the experiment. Ear cells were isolated and loaded with FDG and stained with an anti-CD31 antibody, as described above. Control CD31 $1^{+}$, $\mathrm{CD} 31^{+} / \mathrm{LacZ}^{-}$, or $\mathrm{CD} 31^{+} / \mathrm{LacZ}^{+}$cells were sorted in a MoFLO cell sorter (Dako). Intracellular staining with anti-BrdU (PE) was carried out using BrdU Flow Kit (BD Biosciences - Pharmingen) following the manufacturer's instructions. Events $\left(6-10 \times 10^{4}\right)$ were acquired on a BD FACScalibur or FACSCanto (BD Biosciences - Immunocytometry Systems) and analyzed using FlowJo software (version 4.6.2; Tree Star Inc.).

Bone marrow transplantation. Untreated iORF74 mice 4 to 6 weeks old $(n=20)$ were lethally irradiated with 2 consecutive doses of 8 and $4 \mathrm{~Gy}$. Whole bone marrow was collected from $\mathrm{GFP}^{+}$donor mice (43), and $5 \times 10^{6}$ cells were transplanted i.v. into lethally irradiated iORF74 recipients. Successful grafting of $\mathrm{GFP}^{+} \mathrm{BM}$ was determined by analysis of peripheral blood 4 weeks after transplantation.

Culture of ear cells in Matrigel. Glass coverslips $(22 \times 22 \mathrm{~mm})$ (Corning Incorporated) were placed at the bottom of 6-well plates and covered with $1 \mathrm{ml}$ of Matrigel (BD Biosciences) diluted 1:2 v/v in DMEM. After perfusion through the left ventricle with sterile PBS, ear cells $\left(10^{6}\right)$ from untreated iORF74/LacZ mice were prepared as described above, layered on top of the Matrigel and cultured in DMEM media supplemented with 20\% FCS and penicillin/streptomycin in the presence or absence of DOX $(10 \mu \mathrm{g} / \mathrm{ml})$. At day 0 and after 4, 10, or 15 days of culture, Matrigel was removed and the adherent cells were stained for LacZ or harvested for mRNA extraction.

$R N A$ extraction and Q-PCR. Total RNA from cultured cells was extracted using the RNeasy Mini Kit (QIAGEN) according to manufacturer's instructions. Reverse transcription was performed from $2 \mu \mathrm{g}$ of RNA. Q-PCR was conducted in duplicates from $25 \mathrm{ng}$ cDNA and with $0.4 \mu \mathrm{M}$ of each primer in a $30 \mu \mathrm{l}$ final reaction volume of $\times 1$ SYBR Green PCR Master Mix (Applied Biosystems). PCR cycling conditions were as follows: 1 cycle of $50^{\circ} \mathrm{C}$ for 2 minutes, 1 cycle of $95^{\circ} \mathrm{C}$ for 10 minutes, 40 cycles of $95^{\circ} \mathrm{C}$ for 15 seconds, and $1 \mathrm{cycle}$ of $60^{\circ} \mathrm{C}$ for 1 minute. Relative expression levels

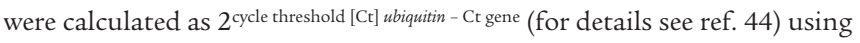
ubiquitin RNA as endogenous control. 
Adoptive transfers. vGPCR/LacZ ${ }^{+}$cells $\left(3 \times 10^{4}\right.$ cells in $\left.20 \mathrm{ml}\right)$ sorted from the ears of iORF74/LacZ mice were injected s.c. using a 31-gauge syringe into the dorsal region of ears and into the dermis of tails of Rag1-deficient recipient mice under anesthesia. After injection, mice were fed ad libitum with regular chow or chow containing 1,000 parts per million (ppm) of DOX (Research Diets Inc.).

In situ bybridization. Animals were deeply anesthetized with sodium pentobarbital $(10 \mathrm{mg} / \mathrm{kg})$. Transcardiac perfusion was performed with $10 \mathrm{ml}$ of phosphate buffer (PB), followed by $30 \mathrm{ml}$ of $4 \%$ PFA in PB at room temperature. Tails were immersion fixed in 4\% PFA in PBS $(10 \mathrm{mM}$ $\mathrm{PB}, 27 \mathrm{mM} \mathrm{KCl}$, and $137 \mathrm{mM} \mathrm{NaCl}, \mathrm{pH} \mathrm{7.4)}$ at $4^{\circ} \mathrm{C}$ for 60 minutes and stored at $30 \%$ sucrose in $\mathrm{PB}$ at $4^{\circ} \mathrm{C}$ for 15 hours. The $10-\mu \mathrm{m}$ cryostat slide-mounted sections were dried immediately under warm air and postfixed in 4\% PFA in PBS for 10 minutes followed by three 10-minute rinses with PBS. Sections were dehydrated for 10 minutes through 70 , 80,90 , and $100 \%$ ethanol. A modified quantum dot FISH protocol was utilized (45). Hybridization was carried out using $1 \mathrm{ng} / \mu \mathrm{l}$ of each biotinylated oligonucleotide probe for $v G P C R$ and for $L a c Z$ or scrambled sequence in buffer containing $\times 4$ standard saline citrate (SSC), $50 \%$ formamide, $0.3 \% \beta$-mercaptoethanol, $100 \mathrm{ng} / \mu \mathrm{l}$ purified BSA, $10 \%$ dextran sulphate, $\times 1$ Denhardt's solution, $1 \mathrm{ng} / \mu \mathrm{l}$ scrambled oligonucleotide probes, $0.3 \%$ Triton $\mathrm{X}-100$, and $0.5 \mathrm{mM}$ vanadyl ribonucleoside complexes. Oligonucleotide sequences available in supplemental material. Following hybridization, the sections were washed 3 times for $30 \mathrm{~min}$ utes each with $\times 1$ SSC at $55^{\circ} \mathrm{C}$ and then for 1 hour with gentle agitation at room temperature. The sections were rinsed once with $\times 0.1 \mathrm{SSC}$ for 5 minutes and then with PBS for 10 minutes. The CdSe/ZnS streptavidinconjugated fluorophore Qdot 565 (Quantum Dot) was diluted to $10 \mathrm{nM}$ in buffer supplied by the manufacturer and incubated for 30 minutes before it was added to the sections for another 30 minutes, followed by 2 10-minute PBS rinses. Nuclear counterstaining was carried out with DAPI $(0.1 \mu \mathrm{g} / \mathrm{ml})$ in PBS for 5 minutes followed by PBS for 5 minutes before coverslipping using glycerol-based mounting medium containing PBS, 90\% glycerol, and 1\% phosphorylated phenylenediamine dihydrochloride (Sigma-Aldrich). Imaging was performed with a Zeiss LSM510META inverted confocal laser scanning microscope using a 40/1.3 NA oil immersion objective. The blue diode laser $405 \mathrm{~nm}$ was used for simultaneous visualization of DAPI and Qdot 565 with bandpass emission filters of 420-480 and 560-615, respectively.

Noncontact laser microdissection. Frozen tail sections were cut $10-\mu \mathrm{m}$ thick, placed on 2.0- $\mu \mathrm{m}$ PEN-membrane slides (Leica Microsystems), and stained for $\beta$-gal activity. The slides were counterstained for 20 minutes with Nuclear Fast Red (Vector Laboratories), and tissue sections were then air dried for 15 minutes at room temperature. Laser microdissection was carried out using the laser microdissection microscope AS LMD (Leica Microsystems) following published procedures (46). Genomic DNA was extracted from more than 2500 individually dissected specimens in each group using the QIAamp DNA Micro kit (QIAGEN) following the manufacturer's instructions. Q-PCR for ORF74, LacZ, neomycin resistance, and ubiquitin genes was performed as described above.

Statistics. For statistical analysis, either 2-tailed unpaired Student's $t$ test or Tukey's test were used. $P$ values less than 0.05 were considered statistically significant.

\section{Acknowledgments}

We are grateful to Jay Unkeless and Paul Frenette for suggestions and critical reading of the manuscript. We thank C.E. Tadokoro for work in the characterization of the iORF74/LacZ mice, Wolfgang Hübner for help with confocal imaging, and Claudia Canasto for excellent technical help. Marcos Grisotto was supported by a grant from the Brazilian Research Council (CNPq -201082/ 2003-9). This work was supported by grants from the NIH (CA109259 to S.A. Lira; U19 AI062623 to S.C. Sealfon) and from the Irene Diamond Fund.

Received for publication August 23, 2005, and accepted in revised form February 7, 2006.

Address correspondence to: Sergio A. Lira, Immunobiology Center, Mount Sinai School of Medicine, 1425 Madison Avenue, Box 1630, New York, New York 10029-6574, USA. Phone: (212) 659-9404; Fax: (212) 849-2525; E-mail: sergio.lira@mssm.edu.
1. Chang, Y., et al. 1994. Identification of herpesviruslike DNA sequences in AIDS-associated Kaposi's sarcoma. Science. 266:1865-1869.

2. Memar, O.M., Rady, P.L., and Tyring, S.K. 1995 Human herpesvirus-8: detection of novel herpesvirus-like DNA sequences in Kaposi's sarcoma and other lesions. J. Mol. Med. 73:603-609.

3. Whitby, D., et al. 1995. Detection of Kaposi sarcoma associated herpesvirus in peripheral blood of HIV-infected individuals and progression to Kaposi's sarcoma. Lancet. 346:799-802.

4. Hengge, U.R., et al. 2002. Update on Kaposi's sarcoma and other HHV8 associated diseases. Part 1: epidemiology, environmental predispositions, clinical manifestations, and therapy. Lancet Infect. Dis. 2:281-292.

5. Sodhi, A., Montaner, S., and Gutkind, J.S. 2004. Does dysregulated expression of a deregulated viral GPCR trigger Kaposi's sarcomagenesis? FASEB J. 18:422-427.

6. Ablashi, D.V., Chatlynne, L.G., Whitman, J.E., Jr., and Cesarman, E. 2002. Spectrum of Kaposi's sarcoma-associated herpesvirus, or human herpesvirus 8, diseases. Clin. Microbiol. Rev. 15:439-464.

7. Dourmishev, L.A., Dourmishev, A.L., Palmeri, D., Schwartz, R.A., and Lukac, D.M. 2003. Molecular genetics of Kaposi's sarcoma-associated herpesvirus (human herpesvirus-8) epidemiology and pathogenesis. Microbiol. Mol. Biol. Rev. 67:175-212.

8. Ganem, D. 1997. KSHV and Kaposi's sarcoma: the end of the beginning? Cell. 91:157-160.

9. Yang, T.Y., et al. 2000. Transgenic expression of the chemokine receptor encoded by human herpesvirus 8 induces an angioproliferative disease resembling Kaposi's sarcoma. J. Exp. Med. 191:445-454.

10. Montaner, S., et al. 2003. Endothelial infection with KSHV genes in vivo reveals that vGPCR initiates Kaposi's sarcomagenesis and can promote the tumorigenic potential of viral latent genes. Cancer Cell. 3:23-36.

11. Guo, H.G., et al. 2003. Kaposi's sarcoma-like tumors in a human herpesvirus 8 ORF74 transgenic mouse. J. Virol. 77:2631-2639.

12. Jensen, K.K., et al. 2005. The human herpes virus 8 -encoded chemokine receptor is required for angioproliferation in a murine model of Kaposi's sarcoma. J. Immunol. 174:3686-3694.

13. Sun, R., et al. 1999. Kinetics of Kaposi's sarcomaassociated herpesvirus gene expression. J. Virol. 73:2232-2242.

14. Chiou, C.J., et al. 2002. Patterns of gene expression and a transactivation function exhibited by the vGCR (ORF74) chemokine receptor protein of Kaposi's sarcoma-associated herpesvirus. J. Virol. 76:3421-3439.

15. Flore, O., et al. 1998. Transformation of primary human endothelial cells by Kaposi's sarcoma-associated herpesvirus. Nature. 394:588-592.

16. Dupin, N., et al. 1999. Distribution of human herpesvirus-8 latently infected cells in Kaposi's sarcoma, multicentric Castleman's disease, and primary effusion lymphoma. Proc. Natl. Acad. Sci. U. S. A. 96:4546-4551.

17. Cesarman, E., Mesri, E.A., and Gershengorn, M.C. 2000. Viral G protein-coupled receptor and Kaposi's sarcoma: a model of paracrine neoplasia? J. Exp. Med. 191:417-422.

18. Glaunsinger, B., and Ganem, D. 2004. Highly selective escape from KSHV-mediated host mRNA shutoff and its implications for viral pathogenesis. J. Exp. Med. 200:391-398.

19. Bubman, D., and Cesarman, E. 2003. Pathogenesis of Kaposi's sarcoma. Hematol. Oncol. Clin. North Am. 17:717-745.

20. Couty, J.P., and Gershengorn, M.C. 2004. Insights into the viral $\mathrm{G}$ protein-coupled receptor encoded by human herpesvirus type 8 (HHV-8). Biol. Cell. 96:349-354.

21. Bais, C., et al. 2003. Kaposi's sarcoma associated herpesvirus $G$ protein-coupled receptor immortalizes human endothelial cells by activation of the VEGF receptor-2/KDR. Cancer Cell. 3:131-143.

22. Simonart, T., Hermans, P., Schandene, L., and Van Vooren, J.P. 2000. Phenotypic characteristics of Kaposi's sarcoma tumour cells derived from patch-, plaque- and nodular-stage lesions: analysis of cell cultures isolated from AIDS and non-AIDS patients and review of the literature. Br. J. Dermatol. 143:557-563.

23. Weninger, W., et al. 1999. Expression of vascular 
endothelial growth factor receptor-3 and podoplanin suggests a lymphatic endothelial cell origin of Kaposi's sarcoma tumor cells. Lab. Invest. 79:243-251.

24. Wang, H.W., et al. 2004. Kaposi sarcoma herpesvirus-induced cellular reprogramming contributes to the lymphatic endothelial gene expression in Kaposi sarcoma. Nat. Genet. 36:687-693.

25. Lee, B., Ratajczak, J., Doms, R.W., Gewirtz, A.M., and Ratajczak, M.Z. 1999. Coreceptor/chemokine receptor expression on human hematopoietic cells: biological implications for human immunodeficiency virus-type 1 infection. Blood. 93:1145-1156.

26. Deichmann, M., Kronenwett, R., and Haas, R. 1997. Expression of the human immunodeficiency virus type- 1 coreceptors CXCR-4 (fusin, LESTR) and CKR-5 in CD34+ hematopoietic progenitor cells. Blood. 89:3522-3528.

27. Li, L., et al. 2003. Nestin expression in hair follicle sheath progenitor cells. Proc. Natl. Acad. Sci. U. S. A. 100:9958-9961.

28. Amoh, Y., et al. 2004. Nascent blood vessels in the skin arise from nestin-expressing hair-follicle cells. Proc. Natl. Acad. Sci. U. S. A. 101:13291-13295.

29. Mokry, J., et al. 2004. Nestin expression by newly formed human blood vessels. Stem Cells Dev. 13:658-664.

30. Amoh, Y., et al. 2005. Hair follicle-derived blood vessels vascularize tumors in skin and are inhibited by Doxorubicin. Cancer Res. 65:2337-2343.

31. Tammela, T., Enholm, B., Alitalo, K., and Paavonen, K.
2005. The biology of vascular endothelial growth factors. Cardiovasc. Res. 65:550-563.

32. Parker, L.H., et al. 2004. The endothelial-cellderived secreted factor Egfl7 regulates vascular tube formation. Nature. 428:754-758.

33. Asahara, T., et al. 1997. Isolation of putative progenitor endothelial cells for angiogenesis. Science. 275:964-967.

34. Shi, Q., et al. 1998. Evidence for circulating bone marrow-derived endothelial cells. Blood. 92:362-367.

35. Kermani, P., et al. 2005. Neurotrophins promote revascularization by local recruitment of $\operatorname{TrkB}$ endothelial cells and systemic mobilization of hematopoietic progenitors. J. Clin. Invest. 115:653-663. doi:10.1172/JCI200522655.

36. Holst, P.J., et al. 2001. Tumorigenesis induced by the HHV8-encoded chemokine receptor requires ligand modulation of high constitutive activity. J. Clin. Invest. 108:1789-1796. doi:10.1172/ JCI200113622.

37. Sodhi, A., et al. 2004. Akt plays a central role in sarcomagenesis induced by Kaposi's sarcoma herpesvirus-encoded $\mathrm{G}$ protein-coupled receptor. Proc. Natl. Acad. Sci. U. S. A. 101:4821-4826.

38. Bais, C., et al. 1998. G-protein-coupled receptor of Kaposi's sarcoma-associated herpesvirus is a viral oncogene and angiogenesis activator. Nature. 391:86-89.

39. Montaner, S., et al. 2004. The small GTPase Rac1 links the Kaposi sarcoma-associated herpesvirus vGPCR to cytokine secretion and paracrine neoplasia.
Blood. 104:2903-2911.

40. Kirshner, J.R., Staskus, K., Haase, A., Lagunoff, M., and Ganem, D. 1999. Expression of the open reading frame 74 (G-protein-coupled receptor) gene of Kaposi's sarcoma (KS)-associated herpesvirus: implications for KS pathogenesis. J. Virol. 73:6006-6014

41. Brown, C.B., et al. 2001. PlexinA2 and semaphorin signaling during cardiac neural crest development. Development. 128:3071-3080.

42. Saaristo, A., et al. 2002. Adenoviral VEGF-C overexpression induces blood vessel enlargement, tortuosity, and leakiness but no sprouting angiogenesis in the skin or mucous membranes. FASEB J. 16:1041-1049.

43. Manfra, D.J., et al. 2001. Leukocytes expressing green fluorescent protein as novel reagents for adoptive cell transfer and bone marrow transplantation studies. Am. J. Pathol. 158:41-47.

44. Applied Biosystems. 1997. User bulletin \#2: ABI PRISM 7700 sequence detection system. http://hcgs. unh.edu/protocol/realtime/UserBulletin2.pdf.

45. Chan, P., Yuen, T., Ruf, F., Gonzalez-Maeso, J., and Sealfon, S.C. 2005. Method for multiplex cellular detection of mRNAs using quantum dot fluorescent in situ hybridization. Nucleic Acids Res. 33:e161.

46. Burbach, G.J., Dehn, D., Del Turco, D., and Deller, T. 2003. Quantification of layer-specific gene expression in the hippocampus: effective use of laser microdissection in combination with quantitative RT-PCR. J. Neurosci. Methods. 131:83-91. 